\title{
Strong Interactions and Gauge-String Duality
}

\author{
Henrique Boschi-Filho and Nelson R. F. Braga \\ Instituto de Física, Universidade Federal do Rio de Janeiro, Caixa Postal 68528, RJ 21941-972 - Brazil
}

(Received on September 15, 2006)

\begin{abstract}
We discuss some recent phenomenological models for strong interactions based on the idea of gauge/string duality. A very good estimate for hadronic masses can be found by placing an infrared cut off in AdS space. Considering static strings in this geometry one can also reproduce the phenomenological Cornell potential for a quark anti-quark pair at zero temperature. Placing static strings in an AdS Schwarzschild space with an infrared cut off one finds a transition from a confining to a deconfining phase at some critical horizon radius (associated with temperature).
\end{abstract}

Keywords: AdS/CFT; String theory; QCD

\section{INTRODUCTION}

In this review we are going to discuss some recent phenomenological results concerning the relation between string theory and QCD inspired in this idea of introducing an infrared cut off in anti-de Sitter (AdS) or in Schwarzschild-AdS spaces. QCD has been tested and confirmed with success in high energy experiments but it is non perturbative at low energies. Lattice calculations give us very important results in this regime. However it seems that we are still far from a complete description of the complexity of strong interactions. In particular, important aspects like confinement and mass generation still lack a satisfactory description. Presently there are many indications that string theory can be useful in the description of strong interactions in the non perturbative regime of QCD.

An early connection between SU(N) gauge theories (for large N) and string theory was realized long ago by 't Hooft[1]. A few years ago a very important result was obtained by Maldacena[2]. He established a correspondence between string theory in $A d S_{5} \times S^{5}$ space-time and $\mathcal{N}=4$ superconformal Yang Mills SU(N) theory for large N at its four dimensional boundary, known as AdS/CFT correspondence[24].

In the AdS/CFT correspondence there is an exact duality between a four dimensional gauge theory and string theory in a ten dimensional space. However, in this formulation, the gauge theory has no energy scale as it is conformal. Although it involves a conformal gauge theory, the AdS/CFT correspondence has been a very important source of inspiration for searching QCD results from string theory. The first idea of breaking conformal invariance in the AdS/CFT context, proposed by Witten, is to consider an AdS Schwarzschild black hole as dual to a non-supersymmetric Yang Mills theory[5]. This approach was used to calculate glueball masses in [612].

\section{QCD SCATTERING AND STRING THEORY}

A very important result relating string theory to the behavior of scattering amplitudes for high energy processes at fixed angles was found by Polchinski and Strassler[13]. In this regime, that corresponds, in terms of Mandelstam variables, to $s \rightarrow \infty$ with $s / t$ fixed, the Veneziano amplitude coming from string theory in flat space shows a soft scattering behavior

$$
A_{\text {Ven. }} \sim \exp \left\{-\alpha^{\prime} s f(\theta)\right\},
$$

where $\theta$ is the scattering angle and $\alpha^{\prime}$ is related to the string tension. In contrast, it was known for a long time that hadronic scattering amplitudes for processes in this regime show a hard scattering behavior, as reproduced by QCD [14, 15]. That means, the amplitudes fall of with a negative power of $s$. Polchinski and Strassler found a solution to this apparent obstacle in the description of strong interactions by string theory considering the duality between gauge theory glueballs and string theory dilatons in an AdS space with an infrared cut off. This way they found the QCD hard scattering behaviour for high energy amplitudes at fixed angles.

The hard scattering behavior was also obtained afterwards in [16] from a mapping between quantum states in AdS space and its boundary found in [17]. We considered an AdS slice as approximately dual to a confining gauge theory. The slice corresponds to the metric

$$
d s^{2}=\frac{R^{2}}{(z)^{2}}\left(d z^{2}+(d \vec{x})^{2}-d t^{2}\right),
$$

with $0 \leq z \leq z_{\max } \sim 1 / \mu$ where $\mu$ is an energy scale chosen as the mass of the lightest glueball. We used a mapping between Fock spaces of a scalar field in AdS space and operators on the four dimensional boundary, defined in [17]. Considering a scattering of two particles into $m$ particles one finds a relation between bulk and boundary scattering amplitudes[16]

$$
S_{\text {Bulk }} \sim S_{\text {Bound. }}\left(\frac{\sqrt{\alpha^{\prime}}}{\mu}\right)^{m+2} K^{(m+2)(1+d)}
$$

where $d$ is the scaling dimension of the boundary operators and $K$ is the boundary momentum scale. This leads to the result for the amplitude

$$
A_{\text {Boundary }} \sim s^{(4-\Delta) / 2},
$$

where $\Delta$ is the total scaling dimension of scattered particles. This reproduces the hard scattering behavior.

For some other results concerning QCD scattering properties from string theory see also [18-24]. 


\section{SCALAR GLUEBALL MASSES}

Using the phenomenological approach of introducing an energy scale by considering an AdS slice we found estimates for scalar glueball mass ratios[25, 26]. In the $\mathrm{AdS}_{5}$ bulk we took dilaton fields satisfying Dirichlet boundary conditions at $z=z_{\max }$

$$
\begin{aligned}
\Phi(z, \vec{x}, t) & =\sum_{p=1}^{\infty} \int \frac{d^{3} k}{(2 \pi)^{3}} \frac{z^{2} J_{2}\left(u_{p} z\right)}{z_{\max } w_{p}(\vec{k}) J_{3}\left(u_{p} z_{\max }\right)} \\
& \times\left\{\mathbf{a}_{p}(\vec{k}) e^{-i w_{p}(\vec{k}) t+i \vec{k} \cdot \vec{x}}+\text { h.c. }\right\}
\end{aligned}
$$

where $w_{p}(\vec{k})=\sqrt{u_{p}^{2}+\vec{k}^{2}}$,

$$
u_{p}=\frac{\chi_{2, p}}{z_{\max }},
$$

is the momentum associated with the $z$ direction and $\chi_{2, p}$ are the zeroes of the Bessel functions: $J_{2}\left(\chi_{2, p}\right)=0$.

On the boundary $(z=0)$ we considered scalar glueball states $J^{P C}=0^{++}$and their excitations $0^{++*}, 0^{++* *}$ with masses $\mu_{p}, p=1,2, \ldots$. Assuming an approximate gauge/string duality the glueball masses are taken proportional to the dilaton discrete modes:

$$
\frac{u_{p}}{\mu_{p}}=\text { const. }
$$

So, the ratios of glueball masses are related to zeroes of the Bessel functions

$$
\frac{\mu_{p}}{\mu_{1}}=\frac{\chi_{2, p}}{\chi_{2,1}}
$$

Note that these ratios are independent of the size of the slice $z_{\text {max }}$. Our estimates are in good agreement with the available lattice[27, 28] and AdS-Schwarzschild [6] results. For a detailed comparison see refs. [25, 26].

For some other results concerning glueball masses using gauge/string duality see for instance [29-33].

\section{HIGHER SPIN STATES AND REGGE TRAJECTORIES}

Recently, very interesting results for the hadronic spectrum were obtained by de Teramond and Brodsky[34] considering scalar, vector and fermionic fields in a sliced $A d S_{5} \times S^{5}$ space. It was proposed that massive bulk states corresponding to fluctuations about the $A d S_{5}$ metric are dual to QCD states with angular momenta (spin) on the four dimensional boundary. This way the spectrum of light baryons and mesons has been reproduced from a holographic dual to QCD inspired by the AdS/CFT correspondence.

We used a similar approach to estimate masses of glueball states with different spins[35]. The motivation was to compare the glueball Regge trajectories with the pomeron trajectories. For soft pomerons [36] experimental results show that the spin $J$ of the pomeron is

$$
J \approx 1.08+0.25 M^{2}
$$

\begin{tabular}{|c|c|c|c|}
\hline $\begin{array}{c}\text { Dirichlet } \\
\text { glueballs }\end{array}$ & $\begin{array}{c}\text { lightest } \\
\text { state }\end{array}$ & $\begin{array}{c}1^{\text {st }} \text { excited } \\
\text { state }\end{array}$ & $\begin{array}{c}2^{\text {nd }} \text { excited } \\
\text { state }\end{array}$ \\
\hline $0^{++}$ & 1.63 & 2.67 & 3.69 \\
$2^{++}$ & 2.41 & 3.51 & 4.56 \\
$4^{++}$ & 3.15 & 4.31 & 5.40 \\
$6^{++}$ & 3.88 & 5.85 & 6.21 \\
$8^{++}$ & 4.59 & 5.85 & 7.00 \\
$10^{++}$ & 5.30 & 6.60 & 7.77 \\
\hline
\end{tabular}

TABLE I: Higher spin glueball masses in $\mathrm{GeV}$ with Dirichlet boundary condition. The value 1.63 is an input from lattice.

\begin{tabular}{|c|c|c|c|}
\hline $\begin{array}{c}\text { Neumann } \\
\text { glueballs }\end{array}$ & $\begin{array}{c}\text { lightest } \\
\text { state }\end{array}$ & $\begin{array}{c}1^{\text {st }} \text { excited } \\
\text { state }\end{array}$ & $\begin{array}{c}2^{\text {nd }} \text { excited } \\
\text { state }\end{array}$ \\
\hline $0^{++}$ & 1.63 & 2.98 & 4.33 \\
$2^{++}$ & 2.54 & 4.06 & 5.47 \\
$4^{++}$ & 3.45 & 5.09 & 6.56 \\
$6^{++}$ & 4.34 & 6.09 & 7.62 \\
$8^{++}$ & 5.23 & 7.08 & 8.66 \\
$10^{++}$ & 6.12 & 8.05 & 9.68 \\
\hline
\end{tabular}

TABLE II: Higher spin glueball masses in $\mathrm{GeV}$ with Neumann boundary condition. The value 1.63 is an input from lattice.

where $M$ is the mass in $\mathrm{GeV}$. It is conjectured that the soft pomerons may be related to glueballs. Recent lattice results are consistent with this interpretation[37].

We assume that massive scalars in the AdS slice with mass $\mu$ are dual to boundary gauge theory states with spin $J$ related by:

$$
(\mu R)^{2}=J(J+4)
$$

We consider both Dirichlet and Neumann boundary conditions and the results for the four dimensional glueball masses with even spin are shown in tables I and II respectively.

We found non linear relations between spin and mass squared. We considered linear approximations representing Regge trajectories

$$
J=\alpha_{0}+\alpha^{\prime} M^{2} .
$$

For Dirichlet boundary conditions, taking the states $J^{++}$with $J=2,4, \ldots, 10$ we found a linear fit with

$$
\alpha^{\prime}=(0.36 \pm 0.02) \mathrm{GeV}^{-2} \quad ; \quad \alpha_{0}=0.32 \pm 0.36
$$

as shown in Figure 1.

For Neumann boundary conditions for the states $J^{++}$with $J=$ $2,4, \ldots, 10$ we found

$$
\alpha^{\prime}=(0.26 \pm 0.02) \mathrm{GeV}^{-2} \quad ; \quad \alpha_{0}=0.80 \pm 0.40,
$$

as shown in Figure 2.

So, comparing these results with eq. (6) we see that Neumann boundary conditions give a glueball trajectory consistent with that of pomerons. These kind of boundary conditions appear in the Randall Sundrum model[38] as a consequence of the orbifold condition. 


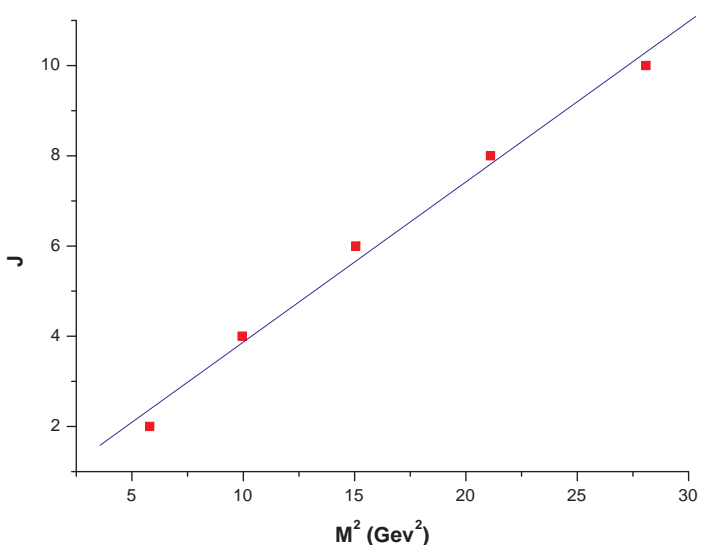

FIG. 1: Spin versus mass squared for the lightest glueball states with Dirichlet boundary conditions from table I. The line corresponds to the linear fit.

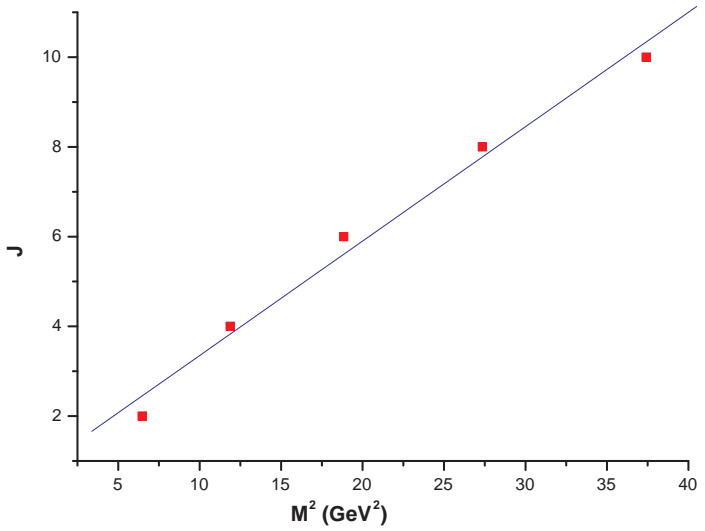

FIG. 2: Spin versus mass squared for the lightest glueball states with Neumann boundary conditions from table II. The line corresponds to the linear fit.

\section{WILSON LOOPS AND QUARK ANTI-QUARK POTENTIAL}

Wilson loops are an important tool to discuss confinement in gauge theories since they show the behavior of the energy associated with a given field configuration. In the AdS/CFT correspondence Wilson loops for a heavy quark anti-quark pair in the gauge theory can be calculated from a static string in the AdS space[39, 40]. The corresponding energy is a non confining Coulomb potential as expected for a conformal theory. For an excellent review and extension to other metrics see [41]. We calculated Wilson loops for a quark anti-quark pair in D3-brane space finding different behaviors, with respect to confinement, depending on the quark position[42].

The static potential energy of a heavy quark anti-quark pair is described by the phenomenological Cornell potential

$$
E_{\text {Cornell }}(L)=-\frac{4}{3} \frac{a}{L}+\sigma L+\text { constant }
$$

where $a=0.39$ and $\sigma=0.182 \mathrm{GeV}^{2}$.

The metric (2) of the AdS space can be rewritten as

$$
d s^{2}=\left(\frac{r^{2}}{R^{2}}\right)\left(-d t^{2}+d \vec{x}^{2}\right)+\left(\frac{R^{2}}{r^{2}}\right) d r^{2}
$$

where $r=R^{2} / z$. We have calculated the energy of a static string in an AdS slice defined by $r_{2} \leq r \leq r_{1}$ [43]. The quark anti-quark pair (string endpoints) is located at $r=r_{1}$, separated by a four dimensional ( $x$ coordinates) distance $L$ and there is an infrared cut off in the space at $r=r_{2}$. From now on we choose $r_{2}=R$. Note that there are two kinds of geodesics, as shown in figure 3 , depending on the value of $L$. For small quark separation $L \leq L_{\text {crit }}$ the geodesics are curve (like curve a ) with one minimum value of the coordinate $r=r_{0}$ which is related to $L$ by

$$
L\left(r_{0}\right)=\frac{2 R^{2}}{r_{0}} I_{1}\left(r_{1} / r_{0}\right)
$$

where $I_{1}(\xi)$ is the elliptic integral

$$
I_{1}(\xi)=\int_{1}^{\xi} \frac{d \rho}{\rho^{2} \sqrt{\rho^{4}-1}} .
$$

The critical value corresponds to $L_{c r i t}=L\left(r_{0}=R\right)$ as in curve b of figure 3 .

The energy for $L \leq L_{\text {crit }}$ can be calculated as

$$
E^{(-)}=\frac{2 R^{2}}{\pi \alpha^{\prime}} \frac{I_{1}\left(r_{1} / r_{0}\right)}{L}\left[I_{2}\left(r_{1} / r_{0}\right)-1\right] .
$$

where $1 / 2 \pi \alpha^{\prime}$ is the string tension and we have subtracted the constant $r_{1} / \pi \alpha^{\prime}$ in such a way that the energy is finite even in the limit $r_{1} \rightarrow \infty$. The integral $I_{2}$ is

$$
I_{2}(\xi)=\int_{1}^{\xi}\left[\frac{\rho^{2}}{\sqrt{\rho^{4}-1}}-1\right] d \rho .
$$

For $L>L_{\text {crit }}$, the geodesics reach the infrared brane as shown in curve $\mathbf{c}$ of figure 3 . The energy can be calculated again subtracting the constant $r_{1} / \pi \alpha^{\prime}$, associated with the quark mass. We obtain

$$
\begin{aligned}
E^{(+)} & =\frac{2 R^{2}}{\pi \alpha^{\prime}} \frac{I_{1}\left(r_{1} / r_{0}\right)}{L}\left[I_{2}\left(r_{1} / r_{0}\right)-I_{2}\left(R / r_{0}\right)\right]-\frac{R}{\pi \alpha^{\prime}} \\
& +\frac{L}{2 \pi \alpha^{\prime}} \frac{I_{1}\left(R / r_{0}\right)}{I_{1}\left(r_{1} / r_{0}\right)},
\end{aligned}
$$




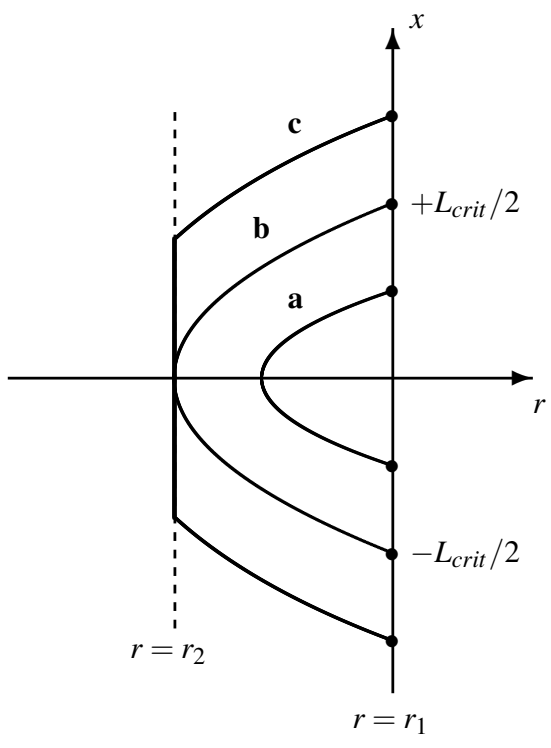

FIG. 3: Schematic representation of geodesics in the AdS slice.

Taking the limit $r_{1} \rightarrow \infty$, we find a potential which is approximately Coulombian for small $L$ and has a leading linear confining behavior for large distances

$$
E^{(+)} \sim \frac{1}{2 \pi \alpha^{\prime}} L
$$

The identification of this potential energy with the Cornell potential leads to

$$
a=3 C_{1}^{2} R^{2} / 2 \pi \alpha^{\prime} ; \quad \sigma=\frac{1}{2 \pi \alpha^{\prime}}
$$

with $C_{1}=\sqrt{2} \pi^{3 / 2} /[\Gamma(1 / 4)]^{2}$. So that we find an effective AdS radius $R=1.4 \mathrm{GeV}^{-1}$. Then the energy takes the form

$$
\begin{aligned}
E^{(-)} & =-\frac{4 a}{3 L}, \quad L \leq L_{c r i t} \\
E^{(+)} & =-\frac{4 a}{3 L}+\frac{4 a}{3 C_{1} L}\left[1-I_{2}\left(R / r_{0}\right)\right] \\
& -\sqrt{\frac{4 a \sigma}{3 C_{1}^{2}}}+\sigma L \frac{I_{1}\left(R / r_{0}\right)}{C_{1}}, \quad L \geq L_{c r i t}
\end{aligned}
$$

The shape of this potential energy is very similar to the Cornell potential as shown in figure 4 .

\section{QUARK ANTI-QUARK POTENTIAL AT FINITE TEMPERATURE}

A gauge/string duality involving a gauge theory at finite temperature was proposed by Witten in [5] inspired by the

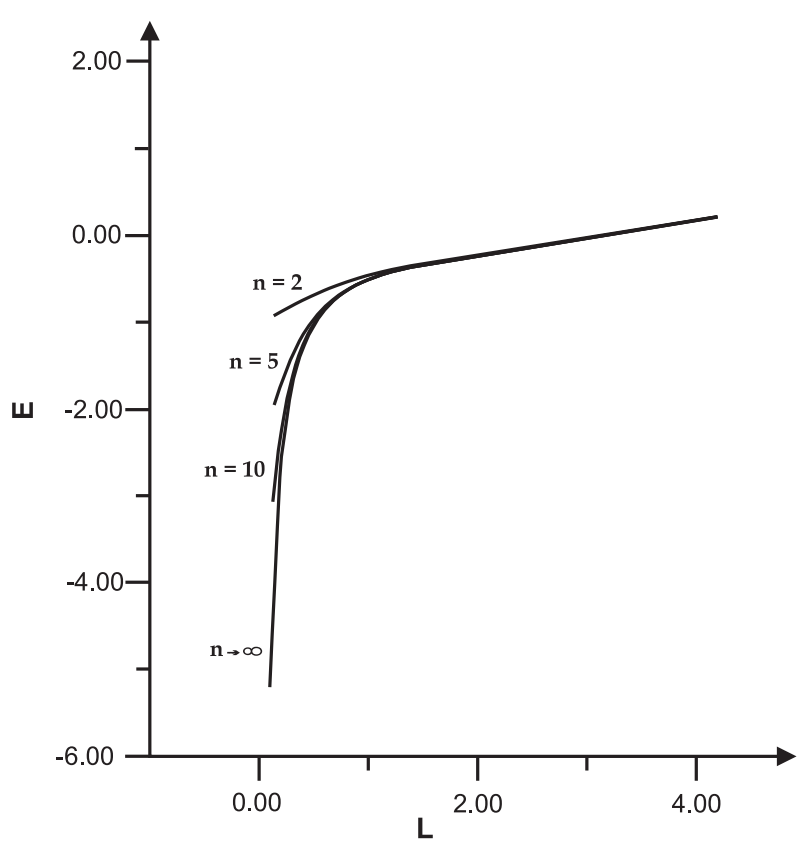

FIG. 4: Energy in $\mathrm{GeV}$ as a function of string end-points separation $L$ in $\mathrm{GeV}^{-1}$, for AdS slices with $r_{1}=n R$ and $r_{2}=R$. For $n \rightarrow \infty$ the energy behaves as the Cornell potential eq. (11).

work of Hawking and Page[44]. In this approach, for high temperatures, the AdS space accommodates a Schwarzschild black hole and the horizon radius is proportional to the temperature. For low temperatures the dual space would be an AdS space with compactified time dimension, known as thermal AdS. The gauge string duality using AdS Schwarzschild space has recently been applied to obtain the viscosity of a quark gluon plasma[45, 46].

In ref. [47] we considered an AdS Schwarzschild black hole metric as a phenomenological model for a space dual to a theory with both mass scale and finite temperature. The corresponding metric is

$$
d s^{2}=\left(\frac{r^{2}}{R^{2}}\right)\left(-f(r) d t^{2}+d \vec{x}^{2}\right)+\left(\frac{R^{2}}{r^{2}}\right) \frac{1}{f(r)} d r^{2}+R^{2} d^{2} \Omega_{5},
$$

where $r_{2} \leq r<\infty, f(r)=1-r_{T}^{4} / r^{4}$ and the horizon radius $r_{T}$ is related to the Hawking temperature by $r_{T}=\pi R^{2} T$. At zero temperature this space becomes an Anti-de Sitter (AdS) slice. The problem of static strings in a space with metric (22), without any cut off, was discussed in detail in[48, 49].

Calculating the energy of static strings in this space we found a deconfinement phase transition at a critical temperature. This transition shows up because, depending on the horizon radius (temperature) relative to the cut off position $r_{2}$, we have different behaviors for the energy. If $r_{T} \geq r_{2}$ the string will not be affected by the presence of the brane since it does not cross the horizon. So the energy will be that described in ref. [48, 49] and there will be no confinement. If $r_{T}<r_{2}$ the energy for large endpoint separation $L$ will grow linearly with $L$ with a temperature dependent coefficient and the quarks are 


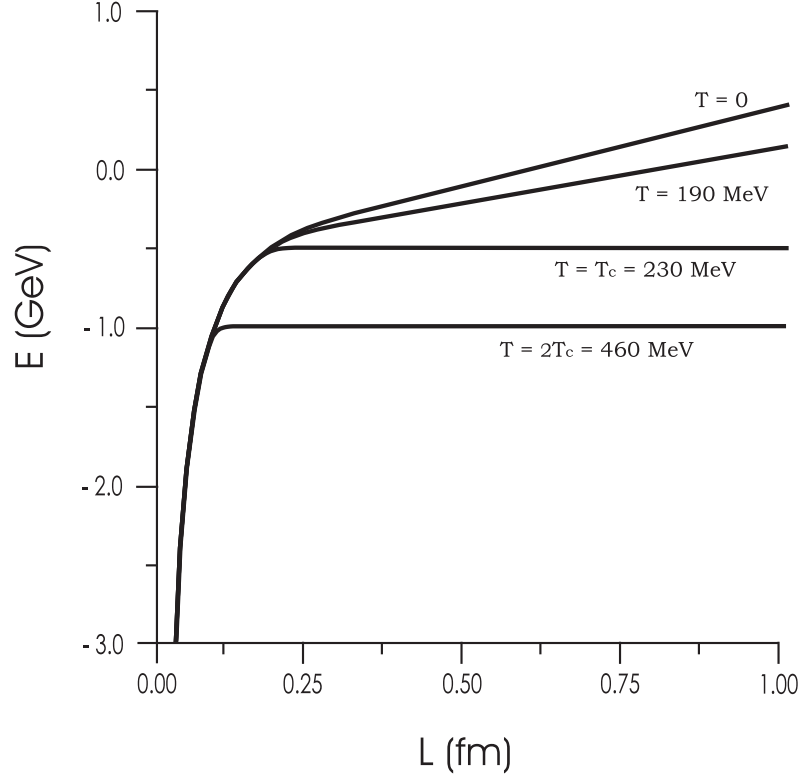

FIG. 5: Energy as a function of string end-points separation for different temperatures.

confined. The critical temperature $T_{C}$ corresponds to $r_{T}=r_{2}$.

As in the zero temperature case we choose $r_{2}=R$ from now on. We show in figure 5 the energies obtained in [47] for temperatures $T=0, T=0.8 T_{C}, T=T_{C}$ and $T=2 T_{C}$. This figure illustrates the fact that in our model the energy of static strings associated with the quark anti-quark potential present a confining behavior for temperatures below $T_{C}=1 / \pi R$. In this case there is a linear term in the energy, for large quark distances $L$ given by $E \sim \sigma(T) L$ with

$$
\sigma(T)=\frac{1}{2 \pi \alpha^{\prime}} \sqrt{1-(\pi R T)^{4}} \quad\left(T<T_{C}\right)
$$

At zero temperature this coefficient is identified with the string tension of the Cornell potential $1 /\left(2 \pi \alpha^{\prime}\right)=0.182 \mathrm{Gev}^{2}$. For temperatures $T \geq T_{C}$ there is no confinement since the energy is finite when $L \rightarrow \infty$. Choosing the brane position to have the value $R=1.4 \mathrm{GeV}^{-1}$ as in the zero temperature case[43] we find a critical temperature $T_{C} \sim 230 \mathrm{MeV}$.

Our results agree qualitatively with lattice calculations for QCD at finite temperature [50-53]. However, it is important to mention that the results from fluctuations of strings in flat space at finite temperature[54] and from lattice calculations[55] imply corrections to the string tension of order $-T^{2}$ at low temperatures, while our model predicts corrections of order $-T^{4}$, as can be seen from eq. (23). If we had considered the thermal AdS metric for low temperatures, instead of the AdS Schwarzschild black hole metric, we would get no thermal corrections to the string tension. It would be interesting to find a holographic phenomenological model that gives the expected low temperature corrections. It is worth mentioning the recent articles [56] and [57] that also discuss thermal effects in the gauge/string duality context. There is also a very recent result by Herzog[58] which indicates that the dual space for temperatures below $T_{C}$ should be the thermal AdS.

For other interesting results concerning gauge/string duality and QCD see for instance [59-69].

\section{Acknowledgments}

We would thank Silvio Sorella and the organizers of "Infrared QCD in Rio", where this talk was presented, for the very warm atmosphere during the workshop. We thank also Michael Teper and Andrei Starinets for very interesting discussions about the finite temperature case. The authors are partially supported by CNPq and Faperj.
[1] G. 't Hooft, Nucl. Phys. B 72, 461 (1974).

[2] J. Maldacena, Adv. Theor. Math. Phys. 2, 231 (1998).

[3] S. S. Gubser, I.R. Klebanov and, A.M. Polyakov, Phys. Lett. B 428, 105 (1998).

[4] E. Witten, Adv. Theor. Math. Phys.2, 253 (1998).

[5] E. Witten, Adv.Theor.Math.Phys. 2, 505 (1998).

[6] C. Csaki, H. Ooguri, Y. Oz, and J. Terning, JHEP 9901, 017 (1999).

[7] R. de Mello Koch, A. Jevicki, M. Mihailescu, and J. P. Nunes, Phys. Rev. D 58, 105009 (1998).

[8] A. Hashimoto, Y. Oz, Nucl. Phys. B 548, 167 (1999).

[9] C. Csaki, Y. Oz, J. Russo, J. Terning, Phys. Rev. D 59, 065012 (1999).

[10] J. A. Minahan, JHEP 9901, 020 (1999).

[11] C. Csaki, J. Terning, AIP Conf. Proc. 494, 321 (1999).

[12] R. C. Brower, S. D. Mathur, and C. I. Tan, Nucl. Phys. B 587, 249 (2000).

[13] J. Polchinski and M. J. Strassler, Phys. Rev. Lett. 88, 031601 (2002).

[14] V. A. Matveev, R. M. Muradian, and A. N. Tavkhelidze, Lett.
Nuovo Cim. 7, 719 (1973).

[15] S. J. Brodsky and G. R. Farrar, Phys. Rev. Lett 31, 1153 (1973); Phys. Rev. D 11, 1309 (1975).

[16] H. Boschi-Filho and N. R. F. Braga, Phys. Lett. B 560232 (2003).

[17] H. Boschi-Filho and N. R. F. Braga, Phys. Lett. B 525, 164 (2002).

[18] S. B. Giddings, Phys. Rev. D 67 , 126001 (2003).

[19] R. C. Brower, C. I. Tan, Nucl. Phys. B 662, 393 (2003).

[20] O. Andreev, Phys. Rev. D 67, 046001 (2003).

[21] J. Polchinski and M. J. Strassler, JHEP 0305 , 012 (2003).

[22] S. J. Brodsky and G. F. de Teramond, Phys. Lett. B 582, 211 (2004).

[23] O. Andreev, Phys. Rev. D 70, 027901 (2004).

[24] O. Andreev, Phys. Rev. D 71, 066006 (2005).

[25] H. Boschi-Filho and N. R. F. Braga, Eur. Phys. J. C 32, 529 (2004).

[26] H. Boschi-Filho and N. R. F. Braga, JHEP 0305, 009 (2003).

[27] C. J. Morningstar and M. Peardon, Phys. Rev. D 56, 4043 (1997). 
[28] M.J. Teper, "Physics from lattice: Glueballs in QCD; topology; SU(N) for all N", arXiv:hep-lat/9711011.

[29] E. Caceres and R. Hernandez, Phys. Lett. B 504, 64 (2001).

[30] R. Apreda, D. E. Crooks, N. Evans, and M. Petrini, JHEP 0405, 065 (2004).

[31] X. Amador and E. Caceres, JHEP 0411, 022 (2004).

[32] M. Schvellinger, JHEP 0409, 057 (2004).

[33] E. Caceres and C. Nunez, JHEP 0509, 027 (2005).

[34] G. F. de Teramond and S. J. Brodsky, Phys. Rev. Lett. 94, 201601 (2005).

[35] H. Boschi-Filho, N. R. F. Braga, and H. L. Carrion, Phys. Rev. D 73, 047901 (2006).

[36] P. V. Landshoff, "Pomerons,", published in "Elastic and Difractive Scattering" Proceedings, Ed. V. Kundrat and P. Zavada, 2002, arXiv:hep-ph/0108156.

[37] H. B. Meyer and M. J. Teper, Phys. Lett. B 605, 344 (2005).

[38] L. Randall and R. Sundrum, Phys. Rev. Lett. 83, 3370 (1999); ibid. 83, 4690 (1999).

[39] S. J. Rey and J. T. Yee, Eur. Phys. J. C 22, 379 (2001).

[40] J. Maldacena, Phys. Rev. Lett. 80, 4859 (1998).

[41] Y. Kinar, E. Schreiber and J. Sonnenschein, Nucl. Phys. B 566, 103 (2000).

[42] H. Boschi-Filho and N. R. F. Braga, JHEP 0503, 051 (2005).

[43] H. Boschi-Filho, N. R. F. Braga, and C. N. Ferreira, Phys. Rev. D 73, 106006 (2006).

[44] S. W. Hawking and D. N. Page, Commun. Math. Phys. 87, 577 (1983).

[45] G. Policastro, D. T. Son, and A. O. Starinets, Phys. Rev. Lett. 87, 081601 (2001)

[46] P. Kovtun, D. T. Son, and A. O. Starinets, Phys. Rev. Lett. 94, 111601 (2005).

[47] H. Boschi-Filho, N. R. F. Braga, and C. N. Ferreira, Phys. Rev. D 74, 086001 (2006). .

[48] S. J. Rey, S. Theisen, and J. T. Yee, Nucl. Phys. B 527, 171 (1998).

[49] A. Brandhuber, N. Itzhaki, J. Sonnenschein, and S. Yankielowicz, Phys. Lett. B 434, 36 (1998).
[50] O. Kaczmarek, F. Karsch, E. Laermann, and M. Lutgemeier, Phys. Rev. D 62, 034021 (2000).

[51] O. Kaczmarek, F. Karsch, P. Petreczky, and F. Zantow, Phys. Lett. B 543, 41 (2002).

[52] O. Kaczmarek, F. Karsch, F. Zantow, and P. Petreczky, Phys. Rev. D 70, 074505 (2004) [Erratum-ibid. D 72, 059903 (2005)].

[53] P. Petreczky, Eur. Phys. J. C 43, 51 (2005).

[54] R. D. Pisarski and O. Alvarez, Phys. Rev. D 26, 3735 (1982).

[55] P. de Forcrand, G. Schierholz, H. Schneider, and M. Teper, Phys. Lett. B 160, 137 (1985).

[56] K. Ghoroku and M. Yahiro, Phys. Rev. D 73, 125010 (2006).

[57] O. Andreev and V. I. Zakharov, "The Spatial String Tension, Thermal Phase Transition, and AdS/QCD," arXiv:hep$\mathrm{ph} / 0607026$.

[58] C. P. Herzog, "A holographic prediction of the deconfinement temperature," arXiv:hep-th/0608151.

[59] R. A. Janik and R. Peschanski, Nucl. Phys. B 625, 279 (2002).

[60] L. A. Pando Zayas, J. Sonnenschein, and D. Vaman, Nucl. Phys. B 682, 3 (2004).

[61] O. Andreev and W. Siegel, Phys. Rev. D 71, 086001 (2005).

[62] F. Bigazzi, A. L. Cotrone, L. Martucci, and L. A. Pando Zayas, Phys. Rev. D 71, 066002 (2005).

[63] J. Erlich, E. Katz, D. T. Son, and M. A. Stephanov, Phys. Rev. Lett. 95, 261602 (2005)

[64] L. Da Rold and A. Pomarol, Nucl. Phys. B 721, 79 (2005).

[65] N. Evans, J. P. Shock, and T. Waterson, Phys. Lett. B 622, 165 (2005).

[66] T. Hambye, B. Hassanain, J. March-Russell, and M. Schvellinger, Phys. Rev. D 74, 026003 (2006).

[67] R. Casero, C. Nunez, and A. Paredes, Phys. Rev. D 73, 086005 (2006).

[68] S. J. Brodsky and G. F. de Teramond, AIP Conf. Proc. 814, 108 (2006). [arXiv:hep-ph/0510240].

[69] S. J. Brodsky and G. F. de Teramond, Phys. Rev. Lett. 96, 201601 (2006). 